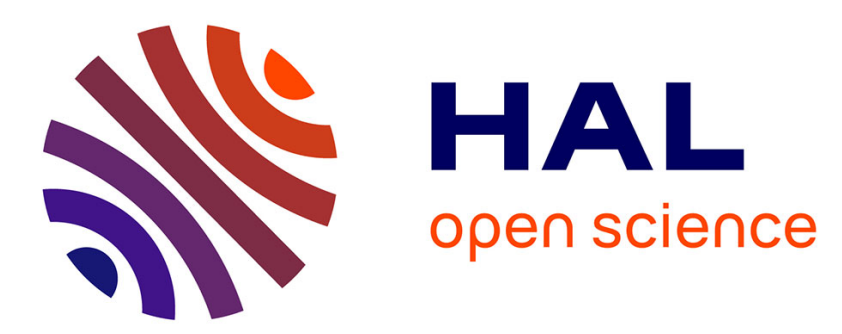

\title{
A new method to introduce constraints in cell-centered Lagrangian schemes
}

\author{
Guillaume Clair, Bruno Després, Emmanuel Labourasse
}

\section{To cite this version:}

Guillaume Clair, Bruno Després, Emmanuel Labourasse. A new method to introduce constraints in cell-centered Lagrangian schemes. Computer Methods in Applied Mechanics and Engineering, 2013, 261/262, pp.56-65. hal-00789201

\section{HAL Id: hal-00789201 https://hal.science/hal-00789201}

Submitted on 17 Feb 2013

HAL is a multi-disciplinary open access archive for the deposit and dissemination of scientific research documents, whether they are published or not. The documents may come from teaching and research institutions in France or abroad, or from public or private research centers.
L'archive ouverte pluridisciplinaire HAL, est destinée au dépôt et à la diffusion de documents scientifiques de niveau recherche, publiés ou non, émanant des établissements d'enseignement et de recherche français ou étrangers, des laboratoires publics ou privés. 


\title{
A new method to introduce constraints in cell-centered Lagrangian schemes
}

\author{
G. Clair ${ }^{\mathrm{a}, 1, *}$ B. Despres ${ }^{\mathrm{b}}$, E. Labourasse ${ }^{\mathrm{a}}$ \\ ${ }^{a} C E A, D A M$, DIF, F-91297 Arpajon, France \\ ${ }^{b}$ UPMC Univ Paris 06, UMR 7598, Laboratoire J.-L. Lions, F-75005 Paris, France
}

\begin{abstract}
We describe a new method to introduce constraints in cell-centered Lagrangian schemes in the framework of compressible hydrodynamics. In this paper we apply it to the modeling of contact and sliding on solid wall boundaries. We illustrate our method, which is based on the minimization of a specific objective function, by several basic problems.
\end{abstract}

Keywords:

Lagrangian Hydrodynamic, Compressible Gas dynamics, Constraints, Minimization method

\section{Introduction}

In this work we describe a new method to solve the Euler's equations of Lagrangian hydrodynamics $[1,2]$ on general unstructured meshes when several constraints apply on the considered mesh. In integral form, the fundamental equations are:

$$
\left\{\begin{array}{lr}
\frac{d}{d t} \int_{V(t)} d V=\int_{V(t)} \nabla \cdot \mathbf{u} d V & \text { volume conservation, } \\
\frac{d}{d t} \int_{V(t)} \rho d V=0 & \text { mass conservation, } \\
\frac{d}{d t} \int_{V(t)} \rho \mathbf{u} d V+\int_{V(t)} \nabla p d V=0 & \text { momentum conservation, } \\
\frac{d}{d t} \int_{V(t)} \rho e d V+\int_{V(t)} \nabla \cdot(p \mathbf{u}) d V=0 \text { total energy conservation. }
\end{array}\right.
$$

Here $\mathrm{V}(\mathrm{t})$ is a volume moving with the fluid and $\frac{D}{D t}=\frac{\partial}{\partial t}+\mathbf{u} \cdot \nabla$ is the material derivative. The physical variables are density $\rho$ (and the specific volume $\tau=1 / \rho$ ), velocity $\mathbf{u}$, total energy e and pressure p.

Constraints we have in mind are encountered in contact and impact problems in the framework of classical mechanics [25, 26], even though the formalism we describe here is

\footnotetext{
*corresponding author: G. Clair

Email address: gclair.recherche@gmail.com (G. Clair)
} 
more general. Such problems deal with interfacial boundaries between non-mixing media (solid, liquid or gas). To analyze specifically interfacial boundaries, numerical simulations are often used. Several contact algorithms have been proposed in the literature: a survey has been recently proposed by N.G. Bourago in the reference [3]. According to the author, contact algorithms can be separated into two major groups, regarding the Lagrangian and Eulerian Motion. The method described in this paper matches with the first category. It is proposed in the framework of Lagrangian cell-centered numerical schemes, for which there is, to our knowledge, no equivalent method that has been published yet (let mention however recent work of Bertoluzza et al. in the context of pure sliding algorithms [4]).

Indeed, the uniqueness of our method is due to the fact that historically, cell-centered Lagrangian schemes are much more recent than staggered schemes. That's why most of contact algorithms have been developed for staggered codes: we cite for instance the contact algorithms in LS-DYNA $[6,7]$ and PRONTO $[8,9,10]$ codes, as well as several contact algorithms based on Lagrange multipliers $[12,13,14,15]$ and penalty function techniques $[16,17]$. This is also the case of the pioneer work of Wilkins about the treatment of sliding lines [18] and most of the sliding line treatments that have followed [19,20]. In cell-centered schemes, two different discrete velocities are used: the first one is cell-centered and satisfies the conservation of momentum. The second one is vertex-centered and is obtained by the solution of a well-posed problem. This structure makes a large difference with staggered methods which have only one instance of the velocity staggered at the vertex, and is one fundamental reason why cell-centered schemes offer potential advantages over staggered methods for the discretization of contact problems.

In this paper, we apply our method to cell-centered Lagrangian schemes of Godunov type (that is fluxes are based on a generalized notion of Riemann invariant), based upon the knowledge of some corner vectors at each time step. The velocities of the $\mathrm{N}$ nodes in the mesh are computed using $\mathrm{N}$ independent $\mathrm{d} \times \mathrm{d}$ linear systems ( $\mathrm{d}$ is the dimension of the considered problem) whose unknowns are the $\mathrm{N}$ nodal velocities of all nodes in the mesh. Such basic formulation is inappropriate to take into account constraints, such as impact and contact, that may apply on several nodes in the mesh. For this purpose, we propose a new formulation of the nodal solver, which is based on a minimization procedure commonly encountered in the modeling of constraints in the solid mechanics community. That is we adopt the framework of constraint optimization which is very natural way of formulating problems with contacts, see $[25,26]$ and references therein. What is new is the coupling with the Riemann solver. Indeed the solution of the multidimensionnal Riemann solver is then seen as the minimum of an objective function within a set of admissible velocities expressing all constraints applying on nodes in the mesh. We prove that this new formulation preserves the main expected properties in the cell-centered Lagrangian schemes that are mass, volume, momentum and total energy conservations. If all constraints are inactive, the new formulation naturally degenerates to the usual method. We stress this paper is dedicated to the abstract framework developed in order to take into account contact and impact, and to first applications to impact and contact between a fluid and a wall. Let emphasize that this method is free from computing the time of impact between the nodes of the mesh and 
the wall: this feature seems to us very pleasant for multidimensional problems for which the exact computation of this time of impact for all nodes and the associated restriction on the time step could generate an important computational burden. This formulation can be extended to the sliding between two fluids, but is beyond the scope of this paper and will be the subject of another paper. While theory is well-suited for 3D implementation, we restrict here to $1 \mathrm{D}$ and $2 \mathrm{D}$ numerical examples.

An outline of this work is as follows. In a first section, we present an overview of traditional nodal solver that are used in cell-centered Lagrangian numerical schemes. We take the example of the GLACE scheme [21], but extension to the EUCCLHYD scheme [5] is easy. Then, we describe the new formulation of the nodal solver. Finally, the efficiency of the method is illustrated with several numerical examples.

\section{Overview of nodal solvers in traditional cell-centered Lagrangian solvers}

\subsection{Introduction of geometrical vector $\boldsymbol{C}_{j, r}$}

Before going further, we remind the notations found in the reference [21] which are needed to define the objective function J. We consider a computational domain $\Omega \subset \mathbb{R}^{d}$ ( $\mathrm{d}$ is the dimension of the problem) composed of control volumes (or cells) indexed by $\mathrm{j}$ and points (or nodes) indexed by $\mathrm{r}$. The volume of the $\mathrm{j}$-th cell at time $\mathrm{t}_{n}$ is denoted $\mathrm{V}_{j}^{n}$ such that:

$$
\bigcup_{j} V_{j}^{n}=\Omega \text { and } \operatorname{dim}\left(V_{i}^{n} \cap V_{j}^{n}\right)<d \text { if } i \neq j .
$$

The set of cells which the r-th node belongs to is denoted $\mathcal{C}(r)$. Similarly, the set of nodes that belong to the $\mathrm{j}$-th cell is denoted $\mathcal{N}(j)$. In addition, we denote respectively $\mathbf{x}_{r}$ and $\mathbf{u}_{r}$ position and velocity vectors of the r-th node within the mesh. In the text, we will often refer to nodal position and nodal velocity.

Let's introduce the corner vectors $\mathbf{C}_{j, r}$ defined as the gradient of the volume $\mathrm{V}_{j}$ with respect to the nodal positions $\mathbf{x}_{r}$ :

$$
\mathbf{C}_{j, r}=\nabla_{\mathbf{x}_{r}} V_{j}
$$

Using this notation, the semi-discretization of the system (1) is the following:

$$
\left\{\begin{aligned}
M_{j} \tau_{j}^{\prime}(t) & =\sum_{r \in \mathcal{C}(r)}\left(\mathbf{C}_{j, r}, \mathbf{u}_{r}\right), \\
M_{j} \mathbf{u}_{j}^{\prime}(t) & =-\sum_{r \in \mathcal{C}(r)} \mathbf{C}_{j, r} p_{j, r}, \\
M_{j} e_{j}^{\prime}(t) & =-\sum_{r \in \mathcal{C}(r)}\left(\mathbf{C}_{j, r}, \mathbf{u}_{r}\right) p_{j, r} .
\end{aligned}\right.
$$

where $\mathbf{u}_{r}$ is the velocity of the $\mathrm{r}$-th node, and $\mathrm{p}_{j, r}$ is a nodal pressure. The latter is computed from a linearized-Riemann-invariant relation in the direction of the $\mathbf{C}_{j, r}$ :

$$
p_{j r}-p_{j}+\rho_{j} c_{j}\left(\mathbf{u}_{r}-\mathbf{u}_{j}, \frac{\mathbf{C}_{j, r}}{\left|\mathbf{C}_{j, r}\right|}\right)=0 .
$$


This relation ensures the entropy property of this family of numerical methods. In the following, we describe the usual method used to compute the nodal velocity $\mathbf{u}_{r}$.

\subsection{Nodal solver}

The nodal velocity is computed by expressing that the sum of all forces around the vertex $\mathbf{x}_{r}$ is zero:

$$
\sum_{j} \mathbf{C}_{j r} p_{j r}=0 .
$$

Substituting the expression of the pressure (3) in the relation (4) leads to solve the system of equations:

$$
\mathbf{A}_{r} \mathbf{u}_{r}=\mathbf{b}_{r} .
$$

where $A_{r}$ and $\mathbf{B}_{r}$ are defined as follow:

$$
\begin{aligned}
\mathbf{A}_{r} & =\sum_{j} \rho_{j} c_{j} \frac{\mathbf{C}_{j, r} \otimes \mathbf{C}_{j r}}{\left|\mathbf{C}_{j, r}\right|}, \\
\mathbf{b}_{r} & =\sum_{j} \mathbf{C}_{j, r} p_{j}+\sum_{j} \rho_{j} c_{j} \frac{\mathbf{C}_{j, r} \otimes \mathbf{C}_{j, r}}{\left|\mathbf{C}_{j, r}\right|} \mathbf{u}_{j} .
\end{aligned}
$$

The matrix $\mathbf{A}_{r}$ is symmetric positive-definite on general meshes, so that the unique solution is:

$$
\mathbf{u}_{r}=\mathbf{A}_{r}^{-1} \mathbf{b}_{r}
$$

\subsection{Boundary conditions for nodal solver}

Usual boundary conditions treatment for cell-centered compressible Lagrangian schemes, as sliding on a planar wall, pressure prescribed on a free surface, given velocity and finally mixed of these conditions at corners, are described in [5, 21, 27]. There is no optimal solutions in the literature for sliding on non planar walls. The method that we propose hereafter to treat more general unilateral boundary conditions can also be used to impose the previous more classical boundary conditions.

\subsection{Formalization of the problem}

Constraints influence the motion of several nodes in the mesh. Therefore, they should be taken into account in the method of computation of the nodal velocities $\mathbf{u}_{r}$. In this sense, solving the system (5) is unadapted. We propose therefore a new formulation based on the minimization of an objective function J under constraints. Before giving more details, we give the following proposition:

Proposition 2.1 (local formulation). Solving the linear system $A_{r} \boldsymbol{u}_{r}=\boldsymbol{b}_{r}, \forall r \in[1: N]$ is equivalent to find within $\mathbb{R}^{d}$ the minimum of the convex quadratic functions $J_{r}$ defined as :

$$
\begin{aligned}
J_{r}: \mathbb{R}^{d} & \rightarrow \mathbb{R} \\
\boldsymbol{u}_{r} & \rightarrow J_{r}\left(\boldsymbol{u}_{r}\right)=\frac{1}{2}\left(A_{r} \boldsymbol{u}_{r}, \boldsymbol{u}_{r}\right)-\left(\boldsymbol{b}_{r}, \boldsymbol{u}_{r}\right) .
\end{aligned}
$$


Proof. $J_{r}$ is well defined over $\mathbb{R}^{d}$. Since $A_{r}$ is a symmetric and positive definite matrix, $J_{r}$ is $\alpha$-convex. Both existence and uniqueness of the global minimum of $J_{r}$ within $\mathbb{R}^{d}$ are ensured by the $\alpha$-convexity of $J_{r}$ (refer for instance to [23]). This minimum is found at the zero of the derivative of $J_{r}$ :

$$
\nabla J_{r}\left(\mathbf{u}_{r}\right)=A_{r} \mathbf{u}_{r}-\mathbf{b}_{r}=0 .
$$

We now describe the new formulation for nodal solvers. Our idea is to minimize an objective function $J$ under constraints, that is looking for the minimum of $J$ in a set of admissible velocities that expresses the constraints. Function $J$ must be a global function of all nodal velocities in the mesh, since, in general, constraints globally apply on the mesh and may couple several nodes. Denoting $\mathbf{U}=\left(\mathbf{u}_{1}, \mathbf{u}_{2}, \ldots, \mathbf{u}_{N}\right)^{T}$ a new velocity vector gathering all nodal velocities in the mesh, our first idea is to define $J$ from the $J_{r}$ functions:

$$
\begin{aligned}
J: \mathbb{R}^{N \times d} & \rightarrow \mathbb{R} \\
\mathbf{U} & \rightarrow J(\mathbf{U})=\sum_{r=1}^{N} \alpha_{r} J_{r}\left(\mathbf{u}_{r}\right) .
\end{aligned}
$$

We suppose $\alpha_{r}>0, \forall r \in[1: N]$, which ensures that $\mathrm{J}$ is a strictly convex quadratic function of $\mathbf{U}$. An important question is to determine the optimal value of the coefficients $\alpha_{r}$. In the section 2.5, we will show that the property of conservation of momentum is preserved with specific values for $\alpha_{r}$. Obviously, we have the following proposition.

Proposition 2.2 (global formulation). For unconstrained problems, solving the local linear systems $A_{r} \boldsymbol{u}_{r}=\boldsymbol{b}_{r}, \forall r \in[1: N]$ is equivalent to find within $\mathbb{R}^{N \times d}$ the minimum of the convex quadratic function $J$ defined by (10).

Proof. J is well defined over $\mathbb{R}^{N \times d}$. J is strictly convex and the convexity of the set $\mathbb{R}^{N \times d}$ then ensures the existence and the uniqueness of a minimum of $J$ within this set. Such minimum is found at the zero of the gradient of $\mathrm{J}$ :

$$
\mathbf{U}_{\min }=\left(\mathbf{u}_{1}, \mathbf{u}_{2}, \ldots, \mathbf{u}_{N}\right)^{T} \text { s.t. } \forall r \in[1: N], \quad \frac{\partial J}{\partial \mathbf{u}_{r}}(\mathbf{U})=\alpha_{r} \nabla_{\mathbf{u}_{r}} J_{r}\left(\mathbf{u}_{r}\right)=\alpha_{r}\left(A_{r} \mathbf{u}_{r}-\mathbf{b}_{r}\right)=0 .
$$

Constraints are taken into account by looking for the minimum of $J_{r}$ within a set of admissible velocities $\mathbb{K} \subset \mathbb{R}^{N \times d}$. In this work, we admit that $\mathbb{K} \neq \emptyset$ and can be written as:

$$
\mathbb{K}=\left\{\mathbf{U} \in \mathbb{R}^{N \times d}, F(\mathbf{U}) \leq 0\right\} .
$$

where $F=\left(F_{1}(\mathbf{U}), F_{2}(\mathbf{U}), \ldots, F_{M}(\mathbf{U})\right)^{T}$ are real functions expressing $M$ constraints applying on all nodes in the mesh. Here $\mathrm{F}(\mathbf{U}) \leq 0$ means that $\mathrm{F}_{j}(\mathbf{U}) \leq 0,1 \leq j \leq M$. We 
will show that this formalism is well adapted to the modeling of contact and impact problems.

For a specific constraint problem, the constrained solution is unique. It means that the minimum of $J$ in $\mathbb{K}$ must exist and be unique. This implies that $\mathbb{K}$ should have the two following properties:

(1) $\mathbb{K}$ is closed, ensuring the existence of the minimum.

(2) $\mathbb{K}$ is convex, ensuring its uniqueness.

This gives the definition of $\mathbf{U}_{\min }$ in the case of constrained problem:

$$
\mathbf{U}_{\min }=\underset{\mathbf{U} \in \mathbb{K}}{\operatorname{argmin}} J(\mathbf{U})
$$

In theory, the method of global minimization must preserve momentum and total energy. For this purpose, $\mathbb{K}$ should have the two additional properties:

(3) Momentum is preserved if translations are admissible solution of any constrained problems. Translations are elements in the form $\mathbf{U}_{\min }+\mathbf{W}_{\mathbf{a}}$, where $\mathbf{W}_{\mathbf{a}}=(\mathbf{a}, \mathbf{a}, \ldots, \mathbf{a})^{T}$, $\forall \mathbf{a} \in \mathbb{R}^{d}$. This property means that the translation of all nodes in the mesh by any vector $\mathbf{a} \in \mathbb{R}^{d}$ naturally preserves the solution of the minimization procedure.

(4) Total energy is preserved if $\mathbb{K}$ is a cone, that is:

$$
\mathbf{U} \in \mathbb{K} \Rightarrow \forall \lambda>0, \quad \lambda \mathbf{U} \in \mathbb{K}
$$

The exact definition of $\mathbb{K}$ will be given for several cases of interest in the section 3 . In particular, the validity of the properties (1), (2), (3) and (4) mentioned above will be discussed for each numerical test case proposed in this paper. We'll see that some of them are sufficient but not necessary.

In the next section, we describe the properties of the method.

\subsection{Properties of the method}

This new formulation ensures the main properties that are expected for the numerical schemes developed in the framework of fluid dynamics: conservation of mass, momentum and total energy. The following propositions give the proof of the two last properties.

Proposition 2.3. If $\mathbb{K}$ has the property (3) and $\alpha_{r}=1$ for all $r \in[1: N]$, the minimization of the objective function $J$ within $\mathbb{K}$ preserves momentum.

Proof. The total momentum variation over a time step writes:

$$
\sum_{j} M_{j} \mathbf{u}_{j}^{\prime}(t)=-\sum_{r}\left(\sum_{j} \mathbf{C}_{j r} p_{j r}\right) .
$$


The minimum of the objective function $J$ satisfies the Euler inequality [23]:

$$
\left(\nabla J\left(\mathbf{U}_{\min }\right), \mathbf{V}-\mathbf{U}_{\min }\right) \geq 0, \forall \mathbf{V} \in \mathbb{K}
$$

The r-th component of $\nabla J\left(\mathbf{U}_{\min }\right)$ writes:

$$
\frac{\partial J}{\partial \mathbf{u}_{r}}\left(\mathbf{U}_{\min }\right)=\alpha_{r} \nabla_{\mathbf{u}_{r}} J_{r}\left(\mathbf{u}_{r}\right) .
$$

The expression of the derivative of $J_{r}$ with respect to $\mathbf{u}_{r}$ is:

$$
\begin{aligned}
\nabla_{\mathbf{u}_{r}} J_{r}\left(\mathbf{u}_{r}\right) & =A_{r} \mathbf{u}_{r}-\mathbf{b}_{r} \\
& =\sum_{j} \rho_{j} c_{j} \mathbf{C}_{j, r} \frac{\left(\mathbf{C}_{j, r}, \mathbf{u}_{r}\right)}{\left|\mathbf{C}_{j, r}\right|}-\sum_{j} \mathbf{C}_{j, r} p_{j}-\sum_{j} \rho_{j} c_{j} \mathbf{C}_{j, r} \frac{\left(\mathbf{C}_{j, r}, \mathbf{u}_{j}\right)}{\left|\mathbf{C}_{j, r}\right|} \\
& =\sum_{j} \mathbf{C}_{j r}\left[-p_{j}+\rho_{j} c_{j}\left(\mathbf{u}_{r}-\mathbf{u}_{j}, \frac{\mathbf{C}_{j, r}}{\left|\mathbf{C}_{j, r}\right|}\right)\right] \\
& =-\sum_{j} \mathbf{C}_{j, r} p_{j, r} .
\end{aligned}
$$

Proof ends by recalling the property (3) of $\mathbb{K}$ : translations are admissible solutions of the constrained problem. This means that, $\forall \mathbf{a} \in \mathbb{R}^{d}$, elements $\mathbf{V}=\mathbf{U}_{\min }+\mathbf{W}_{\mathbf{a}}$ where $\mathbf{W}_{\mathbf{a}}=(\mathbf{a}, \mathbf{a}, \ldots, \mathbf{a})^{T}$, and $\mathbf{V}=\mathbf{U}_{\text {min }}+\mathbf{W}_{\text {-a }}$, where $\mathbf{W}_{\text {-a }}=(\mathbf{- a}, \mathbf{- a}, \ldots, \mathbf{- a})^{T}$, are in $\mathbb{K}$. Thus, inequality (14) becomes the equality:

$$
\begin{array}{cc}
\left(\nabla J\left(\mathbf{U}_{\min }\right), \mathbf{W}_{\mathbf{a}}\right)=0 & \forall \mathbf{a} \in \mathbb{R}^{2}, \\
\Leftrightarrow\left(-\sum_{r} \alpha_{r} \sum_{j} \mathbf{C}_{j, r} p_{j, r}\right) \mathbf{a}=0 \quad \forall \mathbf{a} \in \mathbb{R}^{2}, \\
\Leftrightarrow \sum_{r} \alpha_{r} \sum_{j} \mathbf{C}_{j, r} p_{j, r}=0 .
\end{array}
$$

From (13), conservation of momentum is ensured by choosing $\alpha_{r}=1, \forall r \in[1: N]$. This ends the proof.

From now on, we take $\alpha_{r}=1, \forall r$, so that $J$ may be written in the more general form:

$$
\begin{aligned}
J: \mathbb{R}^{N \times d} & \rightarrow \mathbb{R} \\
\mathbf{U} & \rightarrow J(\mathbf{U})=\frac{1}{2}(A \mathbf{U}, \mathbf{U})-(\mathbf{B}, \mathbf{U}) .
\end{aligned}
$$

where $\quad A=\left(\begin{array}{ccccc}A_{1} & & \ldots & & 0 \\ & \ddots & & & \\ \vdots & & A_{r} & & \vdots \\ & & & \ddots & \\ 0 & & \ldots & & A_{N}\end{array}\right) \quad$ and $\quad \mathbf{B}=\left(\begin{array}{c}\mathbf{b}_{1} \\ \vdots \\ \mathbf{b}_{r} \\ \vdots \\ \mathbf{b}_{N}\end{array}\right)$. 
Next, we show that total energy is preserved.

Proposition 2.4. Assuming moreover that $\mathbb{K}$ has the property (4), then total energy is preserved

Proof. Total energy variation over a time step writes:

$$
\sum_{j} M_{j} e_{j}^{\prime}(t)=-\sum_{r}\left(\sum_{j}\left(\mathbf{C}_{j, r}, \mathbf{u}_{r}\right) p_{j, r}\right)=\left(\nabla J\left(\mathbf{U}_{\min }\right), \mathbf{U}_{\min }\right) .
$$

Again, the proof is based on the Euler inequality:

$$
\left(\nabla J\left(\mathbf{U}_{\min }\right), \mathbf{V}-\mathbf{U}_{\min }\right) \geq 0 \quad \forall \mathbf{V} \in \mathbb{K} \text {. }
$$

$\mathbb{K}$ is a cone, that means we can find two elements $\mathbf{V}_{1}$ and $\mathbf{V}_{2}$ that are written $\mathbf{V}_{1}=$ $(1+\mu) \mathbf{U}_{\min }$ and $\mathbf{V}_{2}=(1-\mu) \mathbf{U}_{\min }$ with $\left.\left.\mu \in\right] 0 ; 1\right]$. Thus, directions $\mu \mathbf{U}_{\min }$ and $-\mu \mathbf{U}_{\min }$ are feasible. From this point, Euler inequality (2.5) turns into equality:

$$
\begin{array}{r}
\left(\nabla J\left(\mathbf{U}_{\text {min }}\right), \mu \mathbf{U}_{\text {min }}\right)=0 \\
\Rightarrow \mu\left(-\sum_{r} \sum_{j}\left(\mathbf{C}_{j, r}, \mathbf{u}_{r}\right) p_{j, r}\right)=0
\end{array}
$$

and, referring to eq. (18), it shows that total energy is preserved.

\subsection{Numerical implementation}

Minimization under constraints is a subject that has been largely described in the literature $[22,23]$. It amounts to introduce a Lagrangian which, as usual, is the sum of the objective function $J$ and of the M constraints applied on the node multiplied by suitable Lagrange multipliers $\boldsymbol{\lambda}=\left(\lambda_{1}, \ldots, \lambda_{M}\right)^{T}$ :

$$
\mathcal{L}(\mathbf{U}, \lambda)=J(\mathbf{U})+\boldsymbol{\lambda} \cdot F(\mathbf{U}) .
$$

In equality-constrained problems, finding $\mathbf{U}$ corresponding to the minimum of $J$ is equivalent to cancel the gradient of the Lagrangian. Assuming now that functions $F$ are now continuously differentiable, we are looking $\mathbf{U} \in \mathbb{K}$ and $\boldsymbol{\lambda} \in \mathbb{R}^{M}$ such as:

$$
\min _{\mathbf{U} \in \mathbb{K}} J(\mathbf{U}) \Longleftrightarrow\left\{\begin{array}{l}
\frac{\partial \mathcal{L}}{\partial \mathbf{U}}=\nabla J(\mathbf{U})+F^{\prime}(\mathbf{U})^{T} \cdot \boldsymbol{\lambda}=0 \\
\frac{\partial \mathcal{L}}{\partial \lambda}=F(\mathbf{U}) \leq 0
\end{array}\right.
$$

where $F(\mathbf{U})^{\prime}=\left(F_{1}^{\prime}(\mathbf{U}), \ldots, F_{M}^{\prime}(\mathbf{U})\right)^{T}$ is the gradient of the function $\mathrm{F}$ with respect to the variable $\mathbf{U}$. 
In inequality-constrained problems, $\mathbf{U}$ and $\boldsymbol{\lambda}$ are respectively searched in $\mathbb{K}$ and $\mathbb{R}_{+}^{M}$. Additional conditions, called constraint qualifications, must be respected in consequence:

$$
\min _{\mathbf{U} \in \mathbb{K}} J(\mathbf{U}) \Longleftrightarrow\left\{\begin{array}{l}
\frac{\partial \mathcal{L}}{\partial \mathbf{U}}=\nabla J(\mathbf{U})+F^{\prime}(\mathbf{U})^{T} \cdot \boldsymbol{\lambda}=0, \\
\boldsymbol{\lambda} \cdot F(\mathbf{U}) \leq 0, \text { with } \lambda_{i} \geq 0, \quad \forall i \in[1: M] .
\end{array}\right.
$$

To solve the system (20) or the system (21), we use the Uzawa method [28] which can be reformulated and implemented with the usual matricial and variational formulations described for example in $[29,25]$.

\section{Numerical examples}

We use explicit schemes with a convenient control of the global time step through the usual CFL constraint $[27,5,21]$. The computation of the time of impact is not necessary. That is why the implementation the method described in this paper is easy. We remark that our algorithm is, in dimension one, exactly the usual one before and after the time of impact.

\subsection{D impact between a fluid and a wall}

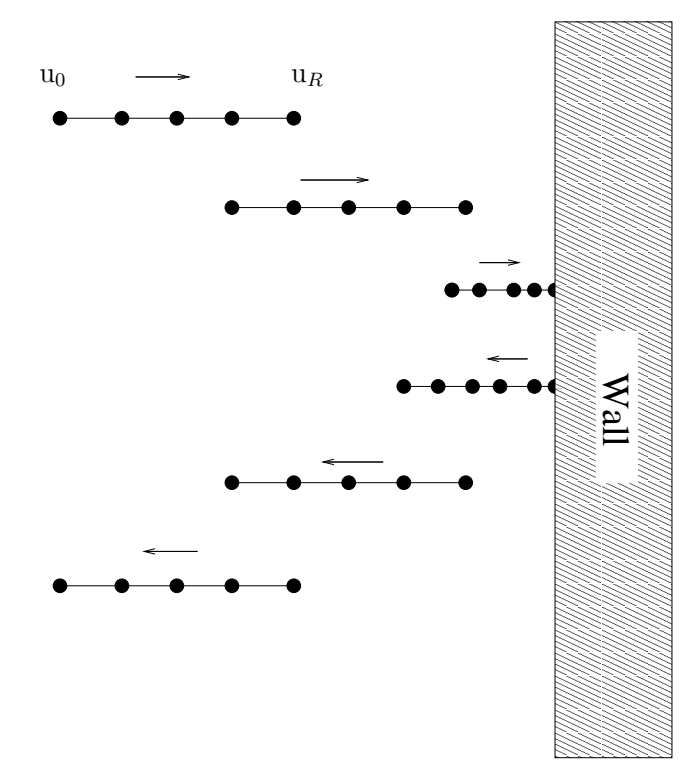

Figure 1: schematics of the fluid-wall 1D impact

The most obvious example is the $1 \mathrm{D}$ contact between a fluid and a wall. Such situation is depicted on figure 1. The surface of the wall is taken as the origin of positions $\mathrm{x}=0$, so the fluid moves in the region $\mathrm{x} \leq 0$. The mesh used for the computation is a regular grid containing $\mathrm{R}=100$ nodes. We define the global velocity vector $\mathbf{U}=\left(u_{1}, u_{2}, \ldots, u_{N}\right)^{T}$. Initially, the fluid of length $\mathrm{L}=1$ is detached from the wall and moves with an initial velocity 
$\mathrm{u}_{0}=1$. Its density is 1 . We choose to work with an ambient pressure equal to 0 , by using a stiffened gas pressure law:

$$
p=(\gamma-1) \rho\left(e-\frac{v^{2}}{2}\right)-\gamma \Pi_{0}
$$

where $\gamma=5 / 3$ and $\Pi_{0}$ is arbitrarily set to $1 / \gamma$.

Let us describe in this example the form of the objective function $J$. We use the general form given by relation (17). A is a diagonal matrix whose diagonal coefficients are written using the relation (6) in the $1 \mathrm{D}$ framework:

$$
\left\{\begin{array}{l}
A_{1}=\rho_{1} c_{1} \\
A_{i}=\left(\rho_{i-1} c_{i-1}+\rho_{i} c_{i}\right) \forall i \in[2: N-1] \\
A_{N}=\rho_{N-1} c_{N-1}
\end{array}\right.
$$

Similarly, the vector $\mathbf{B}$ is written using the relation (7):

$$
\left\{\begin{array}{l}
B_{1}=-p_{1}+\rho_{1} c_{1} u_{1} \\
B_{i}=\left(p_{i-1}-p_{i}\right)+\rho_{i-1} c_{i-1} u_{i-1}+\rho_{i} c_{i} u_{i} \forall i \in[2: N-1] \\
B_{N}=p_{N-1}+\rho_{N-1} c_{N-1} u_{N-1} .
\end{array}\right.
$$

The fluid must remain in the subset $\mathbb{R}_{-}$, so every point must respect the condition:

$$
x(t) \leq 0, \quad \forall t>0 .
$$

The discrete form of the previous relation writes:

$$
\begin{aligned}
x_{r}^{n+1} \leq 0 \quad \forall(r, n) \in[1: N] \times \mathbb{N} \\
\Leftrightarrow x_{r}^{n}+\Delta t u_{r}^{n} \leq 0 \quad \forall(r, n) \in[1: N] \times \mathbb{N}, \\
\Leftrightarrow u_{r}^{n} \leq-\frac{x_{r}^{n}}{\Delta t}, \quad \forall(r, n)[1: N] \times \in \mathbb{N} .
\end{aligned}
$$

In practice, constraint will only be active for the node that impacts the wall, that is the boundary node at the extreme right of the mesh (velocity $u_{N}$ ). Thus, relation (22) always remains a strict inequality for $\mathrm{j} \in[1: N-1]$, and the constraint may be simply written:

$$
u_{N}^{n} \leq-\frac{x_{N}^{n}}{\Delta t}, \quad \forall n \in \mathbb{N}
$$

The set of admissible velocities then writes

$$
\mathbb{K}_{n}=\left\{\mathbf{U} \in \mathbb{R}^{N}, u_{N} \leq-\frac{x_{N}^{n}}{\Delta t}\right\}
$$


(a)

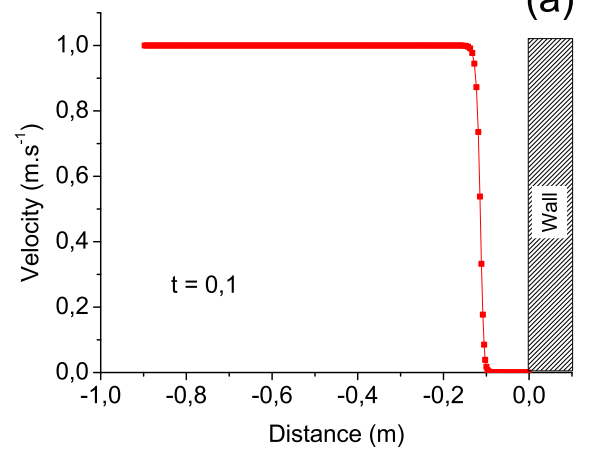

(c)

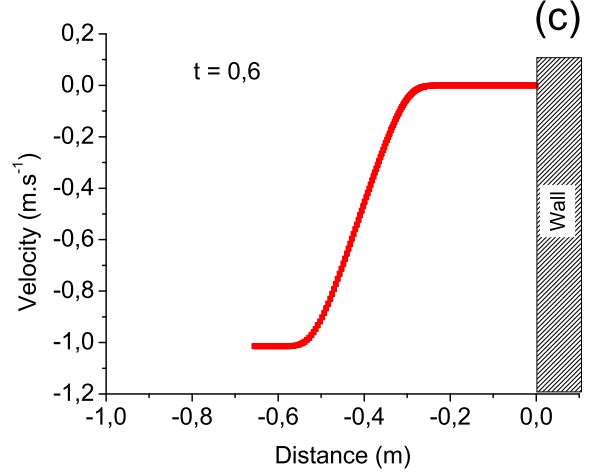

(b)

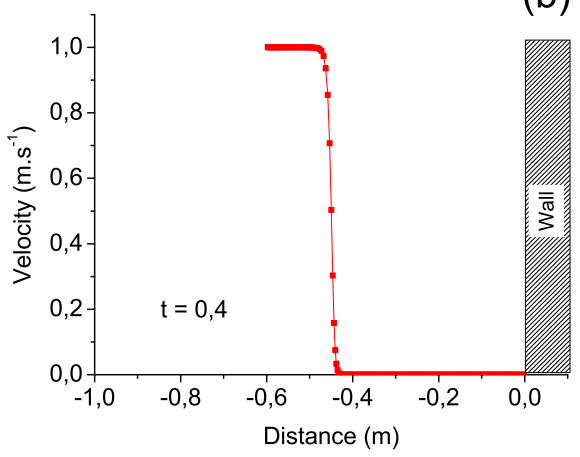

(d)

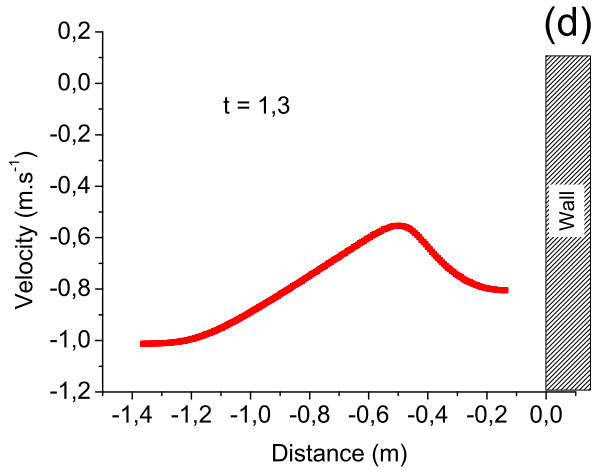

Figure 2: Computed velocity at $\mathrm{t}=0.1(\mathrm{a}), \mathrm{t}=0.4(\mathrm{~b}), \mathrm{t}=0.6(\mathrm{c})$ and $\mathrm{t}=1.3(\mathrm{~d})$

and the function $F$ writes $(M=1)$

$$
F(\mathbf{U})=u_{N}^{n}+\frac{x_{N}^{n}}{\Delta t}
$$

During the impact, $x_{N}^{n}=0$ and the constraint simplifies to $u_{N} \leq 0$.

Figure 2 presents results about the velocity of the fluid at four different times of computation. Figure 2(a) illustrates the time $t=0.1$ at which impact has already occurred. The constraint is active on the node impacting the wall, forcing the fluid to stop at the wall. The latter enters in a compression phase: the kinetic energy is turned into internal energy, inducing a decrease in the velocity value from 1 to 0 in each cell, and an increase in the pressure. This induces the formation of a left-running shock wave, that may be observed at $\mathrm{x} \approx-0.1$ on the part (a) of the velocity profile. On Figure 2(b), shock wave is going to reach the left boundary. After that, the fluids enter in an expansion phase, during which the internal energy turns into kinetic energy. The velocity decreases from 0 to a negative value in each cell. The drop in pressure results in the formation of a rarefaction fan that moves to the right on Figure 2(c). When the velocity of the constrained node becomes negative (the constraint becomes inactive for $\left.u_{N}<0\right)$, the fluid detaches from the wall, as seen on Figure 2(d).

Let us check the properties of $\mathbb{K}_{n}$ in this numerical test case: 
1. $\mathbb{K}$ is closed.

2. $\mathbb{K}$ is convex.

3. When impact occurs, and as long as the fluid remains attached to the wall, translations in the $\mathrm{x}$-positive direction are not admissible solution of the constrained problem. In others words, we can't find any element in $\mathbb{K}_{n}$ in the form $\mathbf{U}_{\min }+\mathbf{W}_{a} \in \mathbb{K}_{n}$, where $\mathbf{W}_{a}=(a, a, \ldots, a)^{T}, a \in \mathbb{R}_{+}^{*}$. Indeed, during the impact, the solution for the N-th component of $\mathbf{U}_{\min }$ is $u_{N}=\frac{-x_{N}^{n}}{\Delta t}$, and we can't find any element $a>0$ that satisfies the constraint:

$$
u_{N}+a \leq \frac{-x_{N}^{n}}{\Delta t}
$$

As a consequence, momentum is not preserved. We can show that it decreases as long as impact occurs. Since directions $\mathbf{W}_{-a}=(-a,-a, \ldots,-a)^{T}, a \in \mathbb{R}_{+}^{*}$ are always feasible, , we can deduce from (14) that:

$$
\begin{aligned}
\left(\nabla J\left(\mathbf{U}_{\min }\right), \mathbf{W}_{-a}\right) & \geq 0 \quad \forall a \in \mathbb{R}_{+}^{*}, \\
\Leftrightarrow\left(-\sum_{r} \sum_{j} \mathbf{C}_{j, r} p_{j, r}, a\right) & \leq 0 .
\end{aligned}
$$

From (13), it shows that momentum decreases during the impact, as expected from mechanical considerations.

On the contrary, when the constraint is inactive, $\mathbf{U}_{\min }$ is the solution of the unconstrained problem, for which $\nabla_{\mathbf{U}} J\left(\mathbf{U}_{\min }\right)=0$. Recalling the expression of the gradient of $J$ from relation (15), and using (13), momentum is then automatically preserved.

4. $\mathbb{K}_{n}$ is a cone when the fluid is attached to the wall. Indeed, in this case, $x_{N}^{n}=0$. The solution of the constraint problem is $u_{N}=0$ and any $\lambda>0$ satisfies the relation $\lambda u_{N} \leq 0$. Thus, total energy is preserved.

However, a change in total energy arises during the time step over which the fluid impacts the surface. In this case, the solution of the constrained problem for the Ncomponent of the velocity is $u_{N}=\frac{-x_{N}^{n}}{\Delta t}>0$. There is no $\lambda>0$ that satisfies the constraint $\lambda u_{N} \leq \frac{-x_{N}^{n}}{\Delta t}$, involving that $\mathbb{K}_{n}$ is not a cone. Going further, there are no elements in the form $(1+\mu) \mathbf{U}_{\text {min }}, \forall \mu>0$ in $\mathbb{K}_{n}$. However, elements in the form $(1-\mu) \mathbf{U}_{\min }, \forall \mu>0$ can be found in $\mathbb{K}_{n}$. Recalling the proof of the proposition 2.4, the Euler can't turn into an equality, and can only be written for the feasible direction $-\mu \mathbf{U}_{\min }$ :

$$
\begin{aligned}
\left(\nabla J\left(\mathbf{U}_{\min }\right),-\mu \mathbf{U}_{\min }\right) & \geq 0, \\
\Leftrightarrow-\mu \sum_{r} \sum_{j}\left(\mathbf{C}_{j, r}, \mathbf{u}_{r}\right) p_{j, r} & \leq 0 .
\end{aligned}
$$


From the relation (18), we have $\sum_{j} M_{j} e_{j}^{\prime}\left(t_{c}\right) \leq 0$, explaining the drop in total energy at the time of impact. We illustrate this loss on the figure 3.

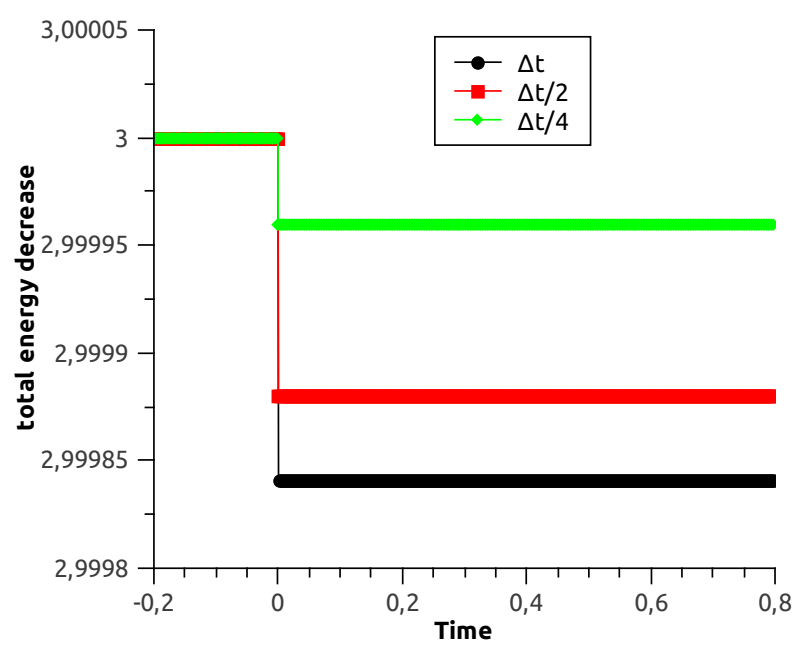

Figure 3: Numerical loss of total energy at the time of impact in the case of 1D impact problem between a fluid and a wall. Results are given for decreasing values of the time step.

A simple expression of the lost amount of energy can be given by writing explicitly the relation $(25)$ :

$$
-\sum_{r} \sum_{j}\left(\mathbf{C}_{j, r}, \mathbf{u}_{r}\right) p_{j, r}=-\left(p_{N-1, N} u_{N}\right) .
$$

Before the impact and when the fluid detaches from the wall, $p_{N-1, N}=0$ since we work with a zero ambient pressure. Total energy is conserved. This is also the case when the fluid is attached to wall, because the constraint imposes $u_{N}=0$. However, over the time step of impact, $u_{N}=\frac{-x_{N}}{\Delta t}>0$ and the relation (3) gives a positive value for $p_{N-1, N}$. Then there is a loss of total energy over this time step. This is illustrated on figure 3 for several time steps.

As long as the fluid is attached to the wall, the situation may be seen as a 1D Noh problem [24], which admits a simple analytical solution. We present therefore a comparison between the latter solution and the numerical solution given by our method. For that purpose, we suppose that the fluid is initially attached to the wall at $\mathrm{x}=0$. The fluid lies in the subset $\mathbb{R}_{\text {- }}$. Initial conditions are uniform in density, $\rho=1$, internal energy $e=0$, and gas velocity $u=1$. In our case, the fluid moves to the right $(u=1)$, while it moves to the left in the original Noh test case $(u=-1)$. The pressure law is a perfect gas law with $\gamma=\frac{5}{3}$.

The exact solution of the Noh problem and the solution given by our method at the time of computation $\mathrm{t}=0.6 \mathrm{~s}$ are presented on figure 4 . The comparison, made for the density and the pressure, shows a good agreement between the numerical and the exact solution. 
The shock front is well captured. On the density profile, the "wall-heating" effect may be evidenced above the wall, which is a common drawback of the Lagrangian schemes.
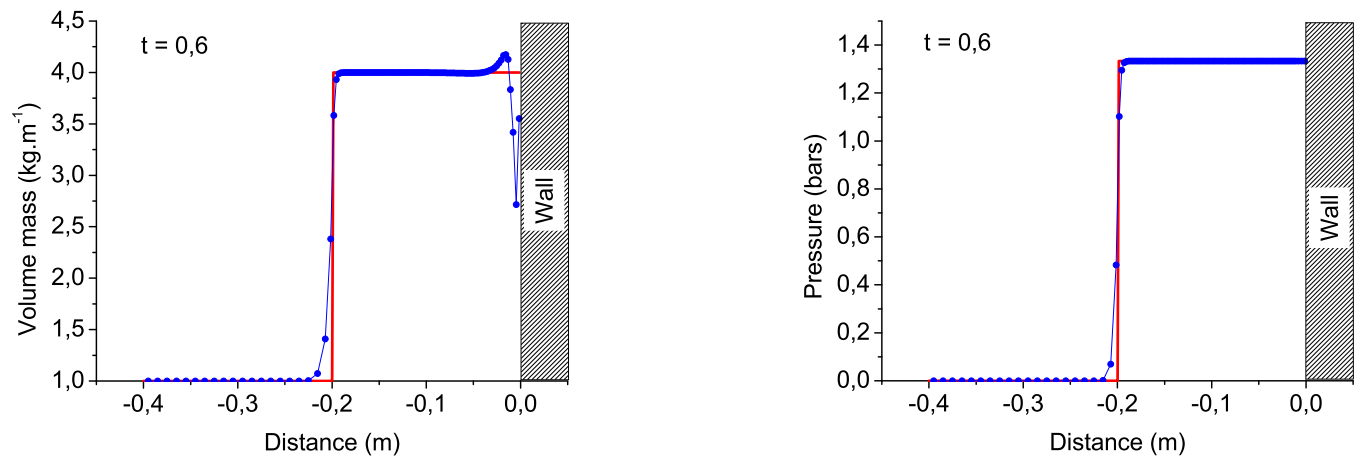

Figure 4: Comparison between the analytical solution of the 1D Noh Problem (line) with our numerical solution for the $1 \mathrm{D}$ impact problem (symbol + line)

\subsection{D Impact between two fluids}

We describe in this part the impact of two fluids with different initial velocity and density. Each fluid has a length $\mathrm{L}=1$ and is meshed with a regular grid with $\mathrm{N}=100$ nodes. Nodes are continuously indexed from 1 to $2 \mathrm{~N}$ : nodes indexed from 1 to $\mathrm{N}$ belong to the fluid on the left, while the ones from $\mathrm{N}+1$ to $2 \mathrm{~N}$ belong to the fluid on the right. Again, we define a global velocity vector $\mathbf{U}=\left(u_{1}, u_{2}, \ldots, u_{2 N}\right)^{T}$.

Initial velocities of the fluids are respectively 1 and -1 . Initial positions of each fluid are chosen such as the impact occurs at $\mathrm{x}=0$. Initial densities are 1. Again the pressure law is a stiffened gas law and the initial ambient pressure is zero.

Velocity constraint applies on both $\mathrm{N}$-th and $(\mathrm{N}+1)$-th nodes, expressing that the distance between them must be greater than or equal to 0 at every time:

$$
\begin{aligned}
& x_{N}^{n+1}-x_{N+1}^{n+1} \leq 0, \\
\Leftrightarrow & x_{N}^{n}+\Delta t u_{N}^{n} \leq x_{N+1}^{n}+\Delta t u_{N+1}^{n}, \\
\Leftrightarrow & u_{N}^{n}-u_{N+1}^{n}-\frac{x_{N+1}^{n}-x_{N}^{n}}{\Delta t} \leq 0 .
\end{aligned}
$$

The set of admissible velocities can be written:

$$
\mathbb{K}_{n}=\left\{\mathbf{U} \in \mathbb{R}^{2 N}, u_{N}-u_{N+1}-\frac{x_{N+1}^{n}-x_{N}^{n}}{\Delta t} \leq 0\right\} .
$$

and the function $F$ writes $(M=1)$

$$
F(\mathbf{U})=u_{N}-u_{N+1}-\frac{x_{N+1}^{n}-x_{N}^{n}}{\Delta t} .
$$


(a)
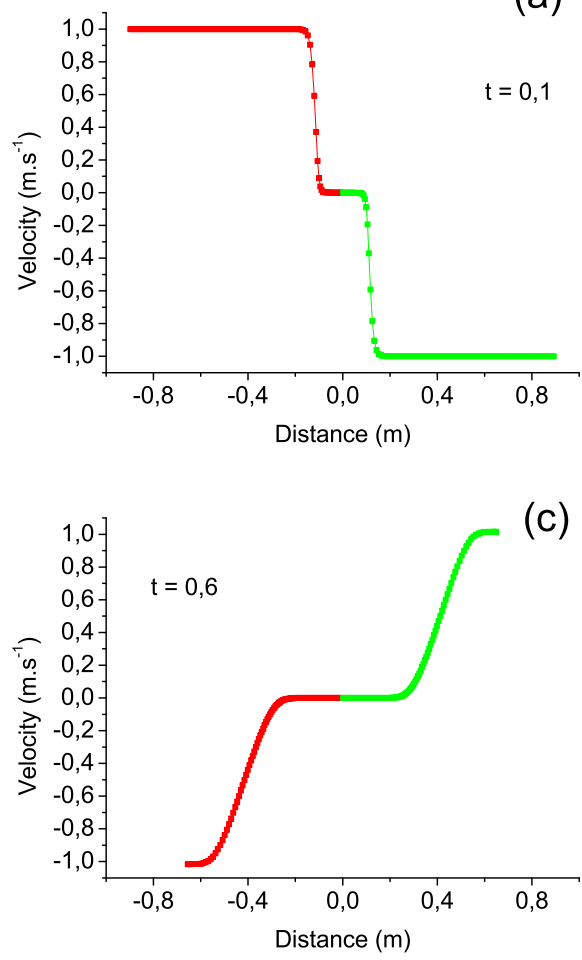

(b)

$\mathrm{t}=0,4$

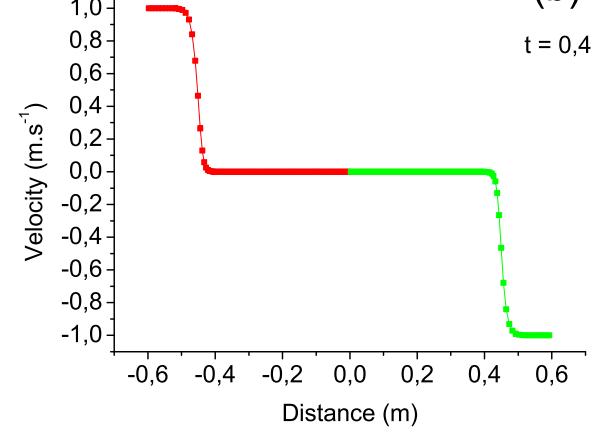

(d)

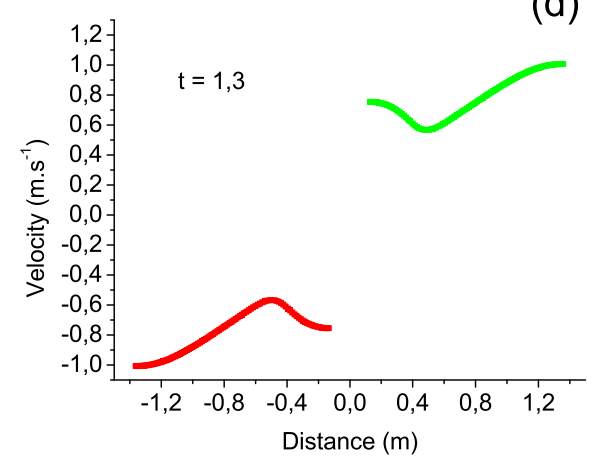

Figure 5: Computed velocity at $\mathrm{t}=0.1(\mathrm{a}), \mathrm{t}=0.4(\mathrm{~b}), \mathrm{t}=0.6(\mathrm{c})$ and $\mathrm{t}=1.3(\mathrm{~d})$

Results about velocity are presented on figure 5 at four different times of computation. One can see that the behavior of both fluids is very similar to the behavior of the fluid in the $1 \mathrm{D}$ problem of the impact on a wall. At $\mathrm{t}=0.1$ (a), both fluids have met, inducing in each one a shock wave that propagates symmetrically to the unconstrained boundary. In the intermediate region, velocity is logically zero by symmetry. When each shock wave reaches the boundary (just after (b)), both fluids have stopped and then expand in the opposite direction of their initial motion. This is observed on figure (c) by the presence of two rarefaction waves propagating in each fluid towards the constrained nodes. The velocity of the constrained node $u_{N}$ becomes negative, while $u_{N+1}$ becomes positive. It leads to the detachment of both fluids, finally moving in opposite directions.

Again, let us examine the validity of properties of $\mathbb{K}_{n}$ in this case. Properties (1) and (2) hold. On the contrary to the previous case, it is obvious that property (3) always holds, since translations by any real $a \in \mathbb{R}^{*}$ are in $\mathbb{K}_{n}$. Thus, momentum is preserved at any time. Let us now discuss about the validity of the property (4). It is clear that the impact between two fluids can be regarded as two separated impact problems for each fluid. Therefore, the same drawbacks that have been described in the case of the impact with a wall will be found in this one. We can show that that total energy is not preserved at the time of impact, due to the fact that $\mathbb{K}_{n}$ is not a cone and there's no element in the form $(1+\mu) \mathbf{U}_{\min }$ in $\mathbb{K}_{n}$, with $\mu \in] 0 ; 1]$. Total energy decreases at the time of impact, which is shown on figure 6 . Again, 
this decrease is $\mathcal{O}(\Delta t)$ for the usual first-order discretization in time of the relation (18).

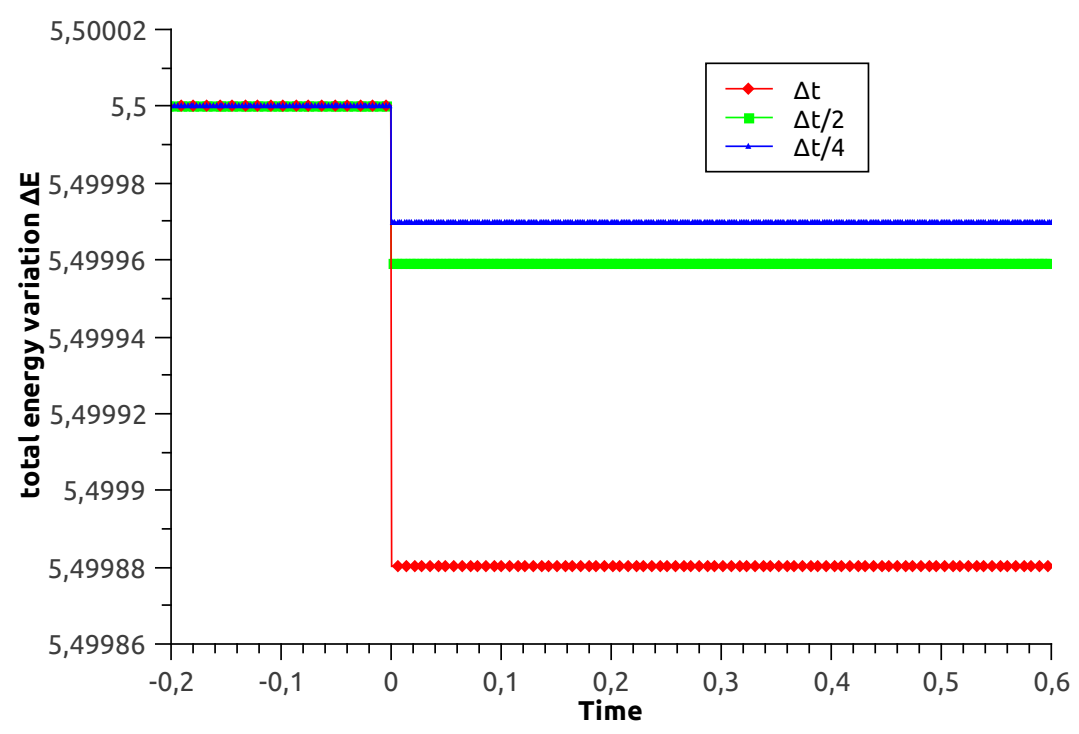

Figure 6: Loss of total energy at the time of impact in the case of 1D Impact Problem between two fluids. Results are given for decreasing values of the time step

\subsection{D Impact of a fluid on a Wall}

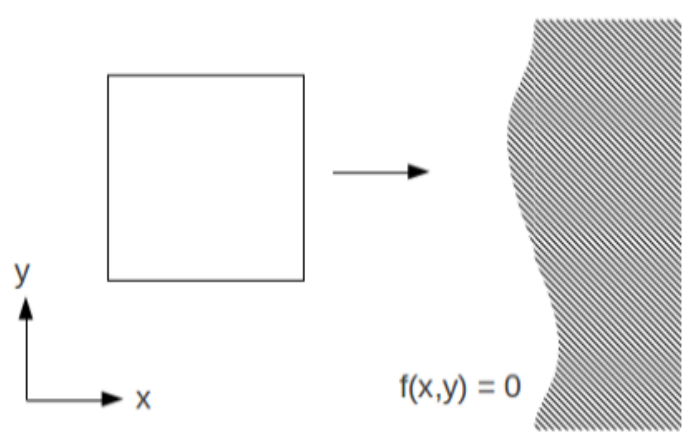

Figure 7: schematics of the fluid-wall impact

Let us describe in this part the extension of the $1 \mathrm{D}$ impact problem to the $2 \mathrm{D}$ framework. The situation is depicted on figure 7 . We admit that the equation of the wall surface has the general form $f(x, y)=0$, so that the fluid initially moves in the subset $f(x, y) \leq 0$. Length and height of the fluid are respectively $\mathrm{L}=1$ and $\mathrm{H}=1$. It is meshed with triangular 


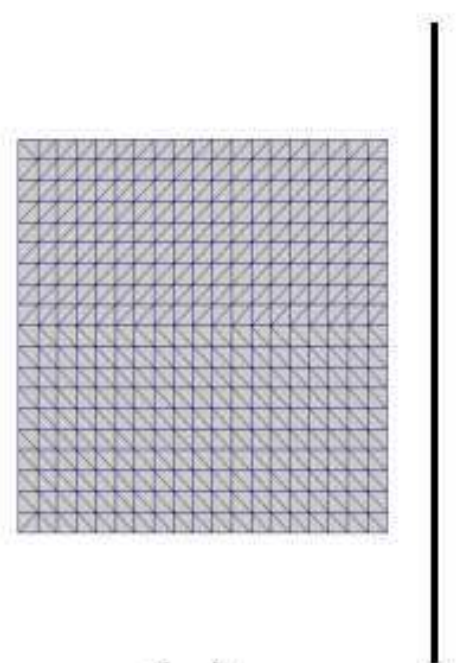

(a)

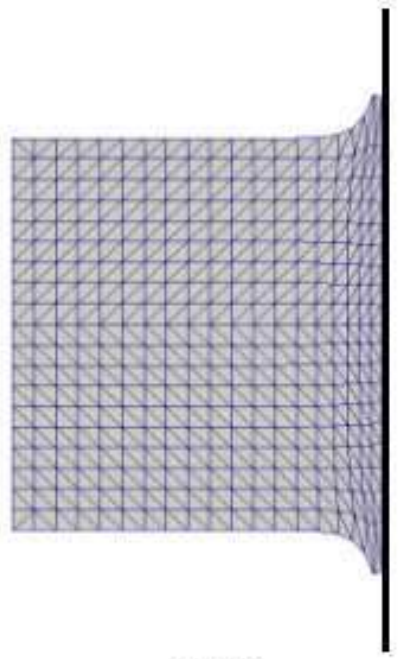

(b)

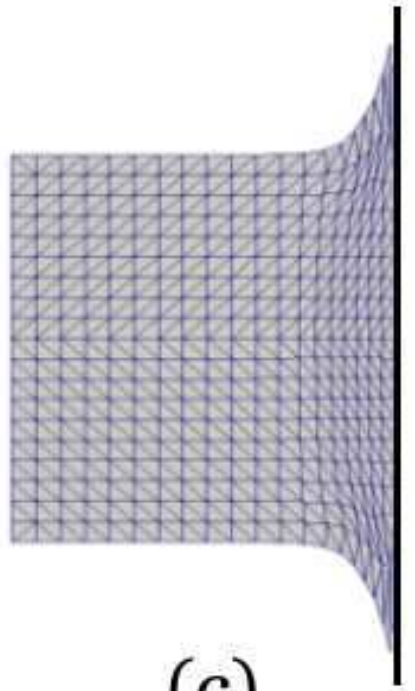

(c)

Figure 8: Fluid impacting on a plane wall. Results refers to three different time of computation (a) $t=0$, (b) $=\mathrm{t}=0.59$ and $(\mathrm{c}) \mathrm{t}=0.67$.

cells for simplicity, and the number of nodes will be denoted N. Again, we define the global velocity vector $\mathbf{U}=\left(\mathbf{u}_{1}, \mathbf{u}_{2}, \ldots, \mathbf{u}_{N}\right)^{T}$ that gathers the velocity vectors of all nodes in the mesh.

The fluid initially moves along the x-direction with a constant velocity equal to 1 . Its density is 1 , the ambient pressure is 0 and we still work with a stiffened gas pressure law. We denote $\mathrm{t}_{c}$ the time of impact.

Constraints apply on every node in the mesh, expressing that their position must remain in the subset $\Omega$ defined as:

$$
\Omega=\left\{(x, y) \in \mathbb{R}^{2}, \quad f(x(t), y(t)) \leq 0 \quad \forall t \geq 0\right\} .
$$

Obviously, constraint for a specific node will be active only if the node impacts the wall. Let us give three examples of $2 \mathrm{D}$ impact problems with different surface geometries. In each case, the properties of $\mathbb{K}$ will be discussed.

\subsubsection{Plane wall}

Let us consider a plan wall orthogonal to the $\mathrm{x}$-direction, whose surface position is located at $x_{\text {surf }}=0$. We denote $\mathbf{x}_{r}=\left(x_{r}, y_{r}\right)^{T}$ and $\mathbf{u}_{r}=\left(u_{r}, v_{r}\right)^{T}$ respectively the position and the velocity vectors of the $r$-th node in the mesh. Recalling the expression (31), the position 
of each node must remain in the subset $f\left(x_{r}(t), y_{r}(t)\right) \leq 0, \forall t \geq 0$. This leads in this particular case to the following discrete formulation of the constraint:

$$
\begin{aligned}
& x_{r}^{n+1} \leq 0 \quad \forall(r, n) \in[1: N] \times \mathbb{N}, \\
\Leftrightarrow & x_{r}^{n}+\Delta t u_{r}^{n} \leq 0 \quad \forall r \in[1: N] \times \mathbb{N}, \\
\Leftrightarrow & u_{r}^{n} \leq \frac{-x_{r}^{n}}{\Delta t} \quad \forall(r, n) \in[1: N] \times \mathbb{N} .
\end{aligned}
$$

The set of admissible velocity $\mathbb{K}_{n}$ is therefore defined as

$$
\mathbb{K}_{n}=\left\{\mathbf{U} \in \mathbb{R}^{2 N}, u_{r} \leq \frac{-x_{r}^{n}}{\Delta t}, \quad \forall r \in[1: N]\right\}
$$

and the functions $F$ write $(M=N)$

$$
F_{r}(\mathbf{U})=u_{r}+\frac{x_{r}^{n}}{\Delta t}, \quad \forall r \in[1: N]
$$

In this case, $\mathbb{K}_{n}$ is obviously closed and convex (properties (1) and (2) holds). When the fluid is attached to the wall, property (3) is violated since translations inducing a motion in the $\mathrm{x}$-positive direction are not allowed. The $\mathrm{x}$-component of the momentum (see eq. (13)) is therefore not preserved. On the contrary, translations inducing a motion in the y-component are admissible, so the y-component of the momentum is preserved. Finally, we can show that the norm of the momentum decreases as long as impact occurs, as well as in the case of the 1D impact between a fluid and a wall.

As long as the fluid is attached to the wall, $\mathbb{K}$ is a cone, and total energy is preserved. Over the time step of impact, the 2D plane case can been seen as $\mathrm{P}$ independent 1D impact problems, where $\mathrm{P}$ is the number of nodes impacting the wall. As a consequence, total energy will decrease as soon as a node impacts the wall. In the plane case, the $\mathrm{P}$ nodes impact the wall at the same time, so that total energy will decrease just over the time step of impact.

Results of our computation are presented on figure 8 at three different times. As expected, we denote that, once the impact has occurred, the x-component $u_{r}$ of the velocity vectors of each node impacting the wall must cancel, while the y-component $v_{r}$ remains non zero. The fluid then slides on the wall.

\subsubsection{Concave wall}

Let us now consider a concave wall whose surface equation is given by the relation:

$$
f(x, y)=x+y^{2}=0 .
$$

In this case this is not only the impact which is challenging but also the treatment of the sliding on such a curve because to our knowledge it is not addressed in the literature in the 


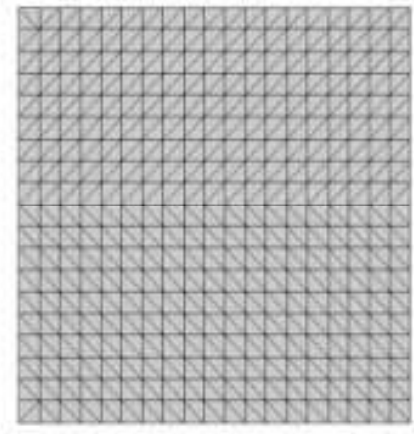

(a)

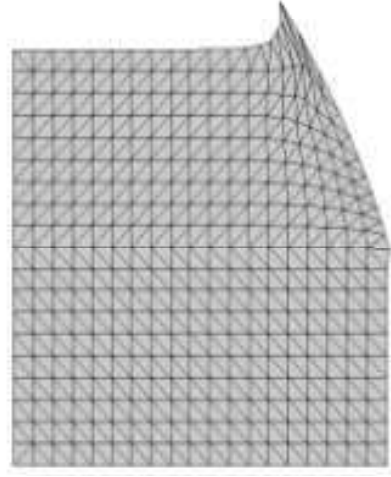

(b)

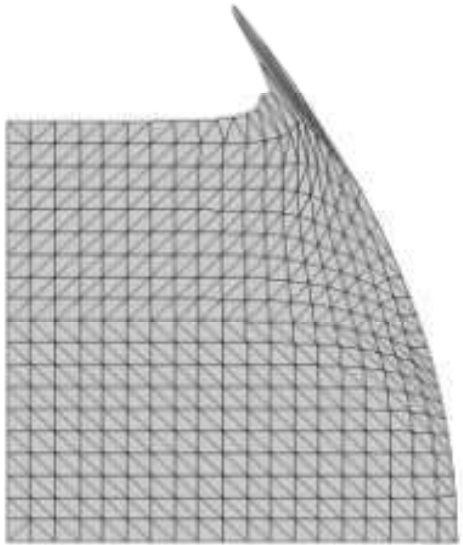

(c)

Figure 9: Fluid impacting on a concave wall whose equation is $x+y^{2}=0$. Results refers to three different time of computation (a) $\mathrm{t}=0,(\mathrm{~b})=\mathrm{t}=0.59$ and $(\mathrm{c}) \mathrm{t}=0.67$.

context of cell-centered Lagrangian schemes. Recalling the condition (31), we directly write the form of the set $\mathbb{K}_{n}$

$$
\mathbb{K}_{n}=\left\{\mathbf{U} \in \mathbb{R}^{2 N}, x_{r}^{n}+\Delta t u_{r}+\left(y_{r}^{n}+\Delta t v_{r}\right)^{2} \leq 0 \quad \forall r \in[1: N]\right\}
$$

and the functions $F$ write $(M=N)$

$$
F_{r}(\mathbf{U})=x_{r}^{n}+\Delta t u_{r}+\left(y_{r}^{n}+\Delta t v_{r}\right)^{2}, \quad \forall r \in[1: N] .
$$

Properties (1) and (2) hold. When the fluid has impacted the wall, properties (3) and (4) do not hold. Property (3) does not hold for the same reason described in the plane case: translations in any direction that induces a penetration of the fluid into the wall are not allowed. Thus, momentum is not preserved and we can show that it decreases as in the plane case.

Property (4) does not hold when impact has occurred. On the contrary to the plane case, nodes impact the wall at different times, inducing for each impact a drop in total energy. Between two consecutive impact, total energy is not preserved since we can't find in $\mathbb{K}$ any element in the form $(1+\mu) \mathbf{U}_{\min }$ with $\mu>0$. To illustrate this argument, we propose the figure 10. Let us consider the $\mathrm{r}$-th node has already impacted the wall and is sliding over it. Let $\mathbf{x}_{r}^{n}$ and $\mathbf{x}_{r}^{n+1}$ be the position of the r-th node at time $t^{n}$ and $t^{n+1} . \mathbf{u}_{r}$ is the r-th component of the vector $\mathbf{U}_{\text {min }}$. Choose any $\left.\left.\lambda \in\right] 0 ; 1\right]$. According to the proposed figure, the 


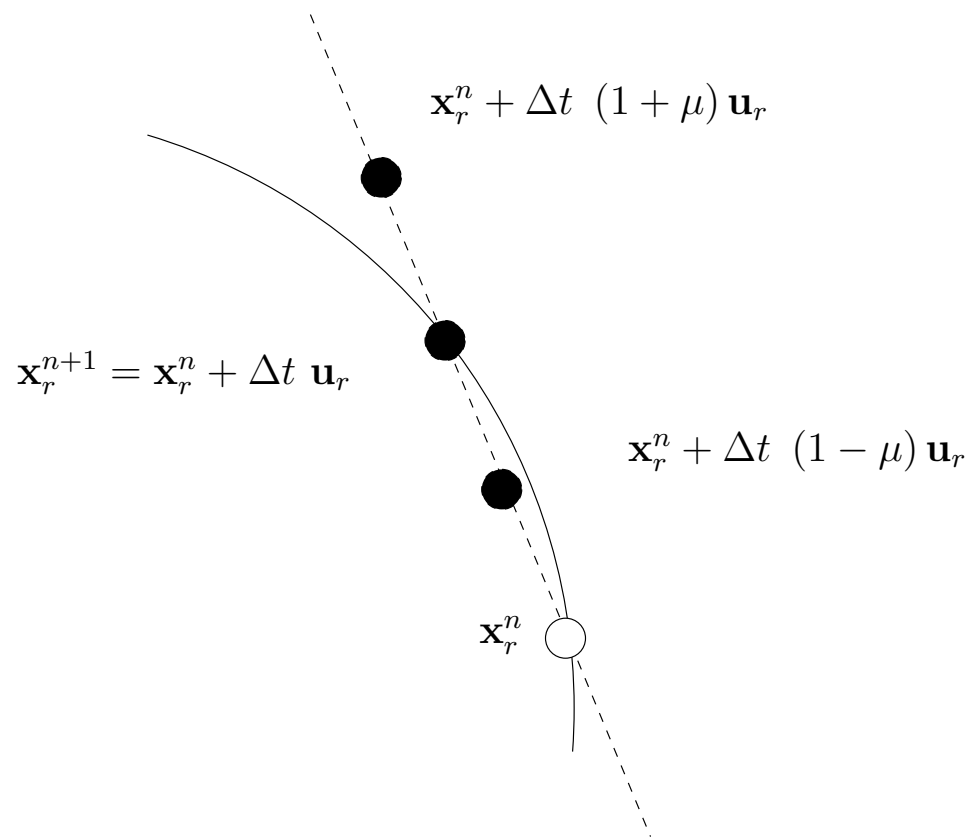

Figure 10: schematics of the fluid-wall 1D impact

velocity $(1-\mu) \mathbf{u}_{r}$ is clearly an element of $\mathbb{K}_{n}$, since it satisfies the constraint (with such velocity, the node would not enter the wall). On the contrary, the velocity $(1+\mu) \mathbf{u}_{r}$ can't be in $\mathbb{K}$ for any $\mu>0$. This argument can be extended to all the nodes impacting the wall, so that there are elements in the form $(1-\mu) \mathbf{U}_{\min }$ in $\mathbb{K}_{n}$ but no elements in the form $(1+\mu) \mathbf{U}_{\text {min }}$, for $\mu>0$. Recalling the proof of the proposition (2.4), the consequence is that total energy decreases between two consecutive impacts. We propose the result on figure 11.

Figure 9 presents results at three different times of computation. As expected, fluid stops at the wall and then slides over it as long at impact occurs. This means that the component of the nodal velocities normal to the wall cancels as long as impact occurs.

\subsubsection{Convex wall}

We consider here a convex wall whose surface equation is given by the relation:

$$
f(x, y)=x-y^{2}=0
$$

The form of the set $\mathbb{K}_{n}$ is

$$
\mathbb{K}_{n}=\left\{\mathbf{U} \in \mathbb{R}^{2 N}, x_{r}^{n}+\Delta t u_{r}-\left(y_{r}^{n}+\Delta t v_{r}\right)^{2} \leq 0 \quad \forall r \in[1: N]\right\}
$$

and the functions $F$ write $(M=N)$

$$
F_{r}(\mathbf{U})=x_{r}^{n}+\Delta t u_{r}-\left(y_{r}^{n}+\Delta t v_{r}\right)^{2} \quad \forall r \in[1: N] .
$$




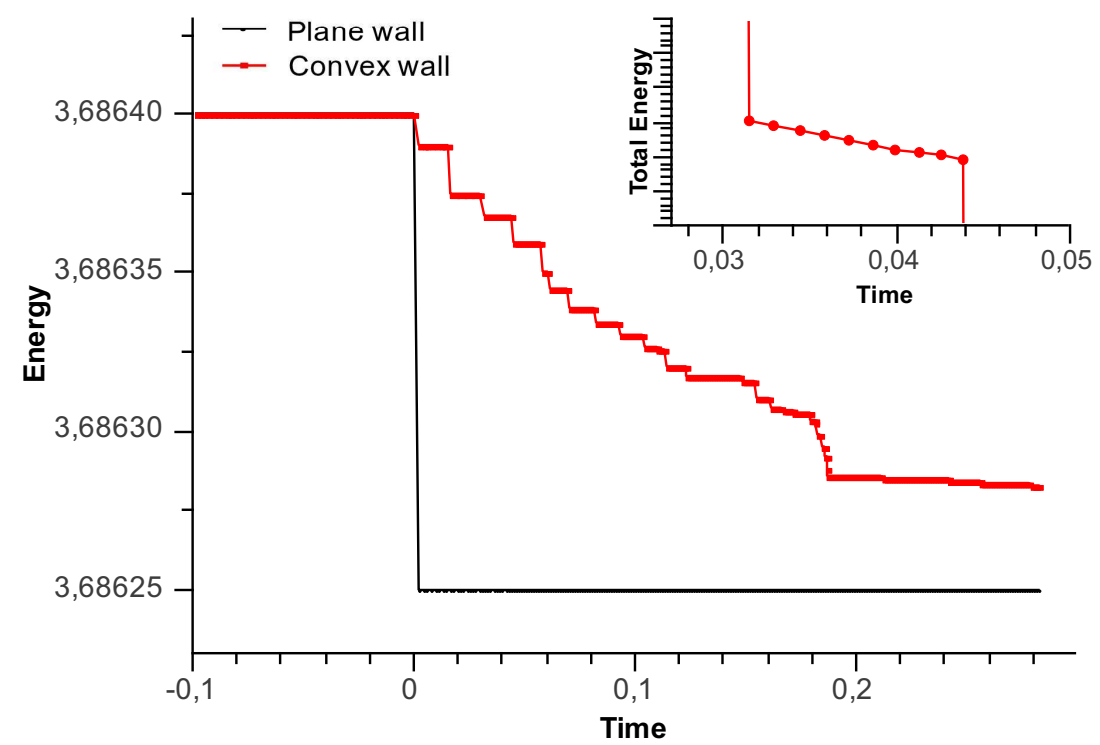

Figure 11: Loss of total energy for 2D impact of a fluid on a wall 


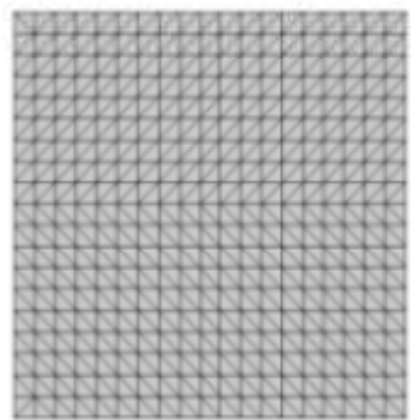

(a)

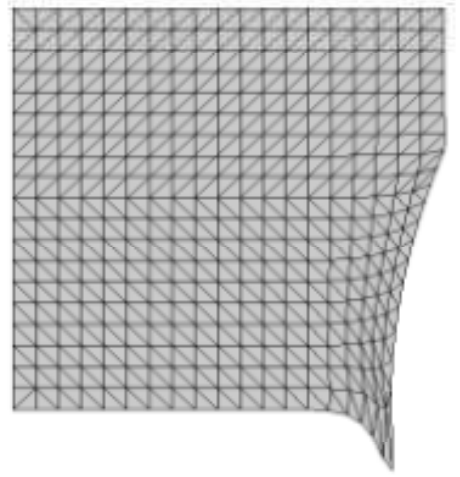

(b)

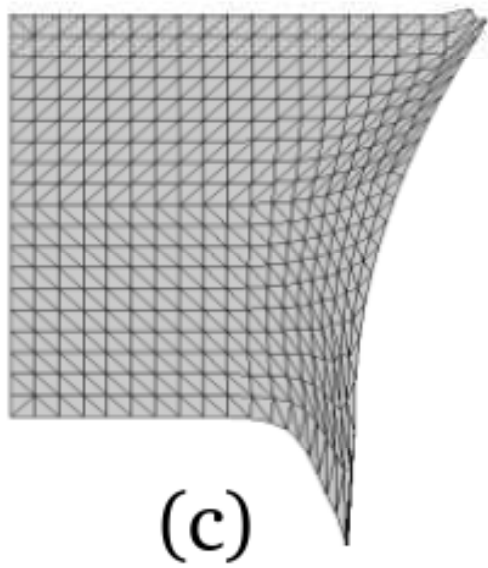

Figure 12: Fluid impacting on a concave wall whose equation is $x-y^{2}=0$. Results refers to three different time of computation (a) $\mathrm{t}=0,(\mathrm{~b})=\mathrm{t}=0.59$ and $(\mathrm{c}) \mathrm{t}=0.67$.

The main difference with the previous case is that the property of convexity (2) of $\mathbb{K}_{n}$ does not hold, which questions the uniqueness of the minimum of the objective function $J$ within $\mathbb{K}_{n}$. Results are proposed on figure 12 . The behavior of the fluid is yet the one expected : while impacting the wall, the fluid slides over it. In this test case, the radius of curvature is sufficiently gentle to ensure that the minimum found in the minimization procedure is the one that corresponds to a correct physical behavior of the fluid. However, if the radius of curvature is much smaller, the minimum may be the wrong one and might lead a priori to the crash of the computation. We illustrate this situation by using the figure 13. We consider a single point impacting a concave wall with a steep radius of curvature. The constraint expresses that the point must not enter the wall. In this case, the position of the point $\mathbf{X}$ may be found within the set $\left.]-\infty ; \mathbf{X}_{A}\right] \cup\left[\mathbf{X}_{B} ;+\infty[\right.$, giving therefore two admissible values for the velocity. The uniqueness of the minimum is then not ensured.

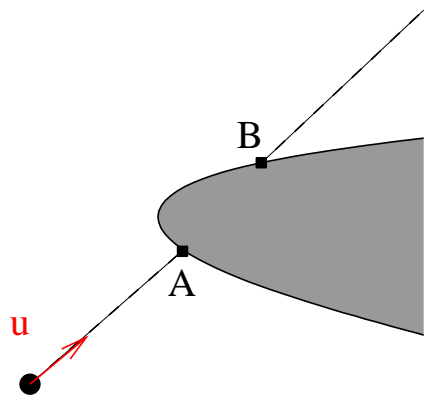

Figure 13: Loss of uniqueness of the minimum of $\mathrm{J}$ within $\mathbb{K}_{n}$ 
However, we think that it is always possible to capture the correct minimum of the objective function $J$ in the case of non convex wall. This is due to the fact that we use an algorithm of minimization (in our case the Uzawa method) that requires an initial estimation $\mathbf{U}_{0}$ to compute the solution $\mathbf{U}_{\min }$. Efficiently, $\mathbf{U}_{0}$ is close to $\mathbf{U}_{\min }$. In our case, we always choose $\mathbf{U}_{0}=\mathbf{U}_{\min }^{n}$ (the solution at the time of computation $t^{n}$ ) to compute the minimum of the objective function $\mathbf{U}_{\min }^{n+1}$ at the time $t^{n+1}$. $\mathbf{U}_{\min }^{n}$ is all the closer to $\mathbf{U}_{\min }^{n+1}$ as the time step $\Delta t$ is small. Thus, by choosing a certain small CFL condition, our method will capture the correct solution even in the case of non convex sets for which several minima of $J$ may exist. This is equivalent to say that there exists an implicit CFL condition that ensures the efficiency of the method. That's what we observe on the figure 12 in the case of a non convex wall.

\section{Conclusions}

We proposed a new formulation of nodal solvers in cell-centered Lagrangian schemes allowing to take into account several types of constraints. Such formulation is based on the minimization of a strictly convex objective function $J$, depending on velocities of the constrained nodes, over a set of admissible velocities $\mathbb{K}$ which expresses constraints applying on the mesh. Four fundamental properties ensure the efficiency of the method. The existence and the uniqueness of the constrained solution $\mathbf{U}_{\min }$ is ensured if $\mathbb{K}$ is a closed set (property (1), and convex (property (2)). The property of convexity is not necessary. We show thanks to an example that the physical solution can be captured for a non-convex set by restricting the CFL condition. Momentum is preserved if $\mathbb{K}$ contains translations (property (3)). Finally, total energy is preserved if $\mathbb{K}$ is a cone (property (4)), but again, such assumption is not necessary : the presence in $\mathbb{K}$ of elements in the form $(1+\mu) \mathbf{U}_{\min }$ and $(1-\mu) \mathbf{U}_{\min }$ for a $\mu>0$, leads to the preservation of total energy. In our numerical tests, the possible loss of energy over the time step of impact, as well as during the impact in the case of curved walls, vanishes for $\Delta t \rightarrow 0$, so that it is not a major problem according to us.

A perspective of our work is the application of the method to the numerical modeling of slide lines in the interior of the computation domain. Slide lines can be expressed as equality constraints, and can be considered as a sub-class of contact problems formulated as inequality or unilateral constraints. Therefore the theory described in this work is convenient to treat slide lines. However, the difficulty results in the discretization of the slip condition to construct $\mathbb{K}_{n}$. This major difficulty will be the subject of a forthcoming paper. Future works will also focus on the 3D extension of the method. While theoretically easy, such issues are practically tedious to implement.

\section{References \\ References}

[1] L.D. Lifschitz, E.M. Landau, Fluid Mechanics (Course of Theoretical physics, Volume $6)$, Second edition (2010) 
[2] H.W. Liepmann, A. Roshko, Elements of Gasdynamics, Dover Publications Inc. (2003)

[3] N. G. Burago, V. N. Kukudzhanov, Mechanics of Solids 40(1), 35-71 (2005)

[4] S. Bertoluzza, S. Del Pino, E. Labourasse, in progress (2013).

[5] P.-H. Maire. J. Comp. Phys., 228(7), 2391-2425 (2009).

[6] J.O. Hallquist, G.L. Goudreau, D.J. Benson, Comput. Methods Appl. Mech. Engrg., 51(1-3), 107-137, 1985

[7] J.O. Hallquist, Report UCRL 52066, Lawrence Livermore Laboratory, Livermore, CA, 1976.

[8] S. W. Attaway, B. A. Hendrickson, S. J. Plimpton, D. R. Gardner, C. T. Vaughan, K. H. Brown, M. W. Heinstein, Computational Mechanics 22, 143-159 (1998)

[9] S. J. Plimpton, S. Attaway, B. Hendrickson, J. Swegle, C. Vaughan, D. Gardner, J. Parallel and Distributed Computing, 50, 104- 122 (1998)

[10] K. Brown, S. Attaway, S. J. Plimpton, B. Hendrickson, Computer Methods in Applied Mechanics and Engineering, 184, 375-390 (2000)

[11] T.J.R. Hughes, R. L. Taylor, J.L. Sackman, A. Curnier, W. Kanoknukulchai, Computer Methods in Applied Mechanics and Engineering, 9, 249-276 (1976).

[12] J.A. González, K.C. Park, C.A. Felippa, R. Abascal, Computer Methods in Applied Mechanics and Engineering, 197(6-8), 623-640 (2008)

[13] M. Tur, F.J. Fuenmayor, P. Wriggers, Comput. Methods Appl. Mech. Engrg., 198(3740), 2860-2873 (2009)

[14] R. Weyler, J. Oliver, T. Sain, J.C. Cante, Comput. Methods Appl. Mech. Engrg., 205208, 68-82 (2012)

[15] G. Zavarise, L. De Lorenzis, Int. J. Numer. Meth. Eng., 91(8), 825-842 (2012)

[16] B. Ruzzeh, J. Kvecses, J. Comput. Nonlinear Dynam. 6(2) 021008-1-12 (2011)

[17] J. Hetherington, A. Rodriguez-Ferran, H. Askes, Int. J. Numer. Meth. Eng., 90(3), 269-286 (2012)

[18] M.L. Wilkins, Computer Simulation of Dynamic Phenomena, Springer (1999)

[19] E.J. Caramana, J. Comp. Phys. 228, 3911-3916 (2009)

[20] M. Kucharík, R. Loubère b, L. Bednárik, R. Liska, to be published in Computers \& Fluids (2012). 
[21] G. Carré, S. Del Pino, B. Després, E. Labourasse, J. Comp. Phys. 228, 5160-5183 (2009)

[22] R. Glowinski, A. Lichnewsky, Computing Methods in Applied Sciences and Engineering, Society for Industrial \& Applied Mathematics, U.S. (1991)

[23] G. Allaire, Numerical Analysis and Optimization: An Introduction to Mathematical Modelling and Numerical Simulation, First edition, OUP Oxford (2007)

[24] M. Gehmeyr , B. Cheng , D. Mihalas, Shock Waves 7, 255-274 (1997)

[25] P. Wriggers, Computational Contact Mechanics, Wiley, 2002.

[26] W.J. Stronge, Impact Mechanics, Cambridge University Press, 2004.

[27] B. Després, C. Mazeran, Lagrangian gas dynamics in 2D and Lagrangian systems, Arch. Rat. Mech. Anal., 178, 2005.

[28] K. Arrow, L. Hurwicz, H. Uzawa, Studies in nonlinear programming, Stanford university press, 1958.

[29] C. Bacuta, A unified approach for uzawa algorithms, Siam Journal Numerical Analysis, Vol. 44, 6, 2633-2649, 2006. 